\title{
Vibration and dynamic response control of cantilevers carrying externally mounted stores
}

\author{
L. Librescu and S. S. Na \\ Engineering Science and Mechanics Department, Virginia Polytechnic Institute \& State University, \\ Blacksburg, Virginia 24061-0219
}

(Received 31 October 1996; accepted for publication 19 August 1997)

\begin{abstract}
The vibrational and dynamic response control of cantilevers carrying externally mounted stores is investigated. The cantilevered structure is modeled as a thin-walled beam of arbitrary cross section and incorporates a number of nonclassical effects such as transverse shear, secondary warping, anisotropy of constituent materials, and heterogeneity of the construction. The control is carried out via a dynamic bending moment applied at the tip of the structure. A feedback control law relating the boundary moment with one of the kinematical variables characterizing the response of the beam is implemented, and its results upon the closed-loop eigenfrequencies and dynamic response are highlighted. The obtained numerical results emphasize the efficiency of this control methodology to enhance, without weight penalties, vibrational and dynamic response behavior and inhibit and even suppress the occurrence of the resonance phenomenon. (c) 1997 Acoustical Society of America.
\end{abstract} [S0001-4966(97)01712-8]

PACS numbers: 43.40.Cw, 43.40.Vn [PJR]

\section{INTRODUCTION}

The cantilever beams carrying externally mounted stores can serve as a basic model for a number of structures used in the aeronautical, aerospace, as well as in other fields of the advanced technology.

Civil and military airplane wings are designed to carry heavy external mounted stores along their span. Depending on their magnitude and location, drastic reduction of natural eigenfrequencies and modification of the eigenmodes are experienced (e.g., see Refs. 1 and 2). These modifications can result in a deterioration of the dynamic response to timedependent excitations and can also precipitate the occurrence of the flutter instability (e.g., see Refs. 3 and 4).

As robot manipulator arms, space booms, or antennas operating in space, they are required to be lightweight, strong, and of high precision in their mission. Uncontrolled vibrations can result in drastic reductions of the accuracy and precision of their operations. In order to be able to control the dynamic response of these structures under timedependent external excitations and inhibit the damaging effects of vibrations without weight penalties, new technologies have to be implemented.

Toward this end, in this paper a number of results related with the vibrational and dynamic response control of cantilevered beams carrying externally mounted stores is addressed. The control mechanism is achieved through the action of a bending moment applied at the tip of the beam. This boundary moment is related, via a linear functional relationship, with the kinematical response quantities, appropriately selected. Such a feedback control methodology was mathematically substantiated quite recently, see, e.g., Refs. 5 and 6. Consistent with the approach in Refs. 7 and 8, in this paper, the implemented feedback control law features a dynamic character. This constitutes a basic departure from the ones in Refs. 9-14, where the adopted control law has a static character and which should be much less efficient than the dynamic one.

In the forthcoming developments, the theory of cantilevers incorporating dynamic feedback control capabilities will be addressed and its implications upon their free vibration and dynamic response will be investigated.

\section{GENERAL CONSIDERATIONS. BASIC ASSUMPTIONS}

The case of the cantilevered thin-walled beams of arbitrary closed cross section is considered. It is assumed that the beam is symmetrically composed of transversely isotropic material layers (the surface of isotropy in each material layer being parallel to the reference surface of the beam structure).

It is assumed that, in addition to a system of concentrated stores of mass $m_{j}$ and mass moment of inertia $I_{j}$ (about their centroid) located at $z=z_{j},(j=\overline{1, J})$, the beam carries also a tip mass of characteristics $M$ and $I_{M}$. In the case of an airplane wing/robot arm the tip mass can simulate the presence of a tank/payload (see Fig. 1). Herein $J$ denotes the total number of wing-mounted stores.

Two systems of coordinates are used in the forthcoming developments, namely: (i) a global Cartesian system $(x, y, z)$, where $(x, y)$ denote the cross-section beam coordinates while the spanwise $z$ axis is assumed to coincide with the locus of symmetrical points of the cross sections along the wing span, and (ii) a local orthogonal coordinate system $(n, s, z)$ where $n$ denotes the thicknesswise coordinate normal to the beam midsurface and $s$ the tangential coordinate along the contour line of the beam cross section.

The equations governing the motion of cantilever beams are established in the context of the following assumptions:

(i) The cross sections of the beam do not deform in their own planes, (ii) The effects of transverse shear flexibility featured by the advanced composite materials are taken into consideration, (iii) The hoop stress resultant $N_{s s}$ is consid- 


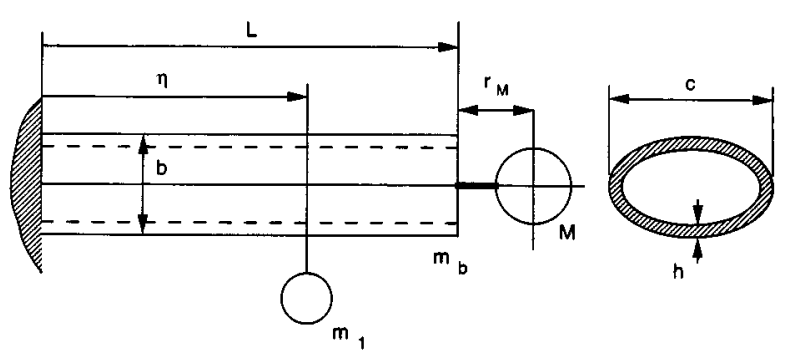

FIG. 1. Geometry of the cantilever beam carrying a unistore and a tip-mass.

ered negligibly small with respect to the remaining ones, (iv) The centroids of the attached masses are distributed along the $z$ axis

As a result of the transverse-isotropy of the constituent materials and in the light of assumption (iv), an exact decoupling of transverse bending (expressed in terms of variables $v_{0}$ and $\theta_{x}$ ), lateral bending (expressed in terms of $u_{0}$ and $\theta_{y}$ ), and twist $(\Theta)$ is obtained. For the problem studied herein only transverse bending will be considered. However, since secondary warping induces transverse bending (See Ref. 16), this effect will also be incorporated.

\section{GOVERNING EQUATION}

Based upon the previously stipulated assumptions, the one-dimensional (1-D) version of the equations governing the flexural motion about the equilibrium position as derived in Refs. 11 and 12 are

$$
\begin{gathered}
a_{55}\left(v_{0}^{\prime \prime}+\theta_{x}^{\prime}\right)-\left[b_{1} \ddot{v}_{0}+\sum_{j=1}^{J} m_{j} \ddot{v}_{0} \delta\left(z-z_{j}\right)\right]+p_{y}=0, \\
a_{33} \theta_{x}^{\prime \prime}-a_{55}\left(v_{0}^{\prime}+\theta_{x}\right) \\
-\left[\left(b_{4}+b_{14}\right) \ddot{\theta}_{x}+\sum_{j=1}^{J} I_{j} \ddot{\theta}_{x} \delta\left(z-z_{j}\right)\right]=0
\end{gathered}
$$

For the beam considered to be clamped at the root and free at the tip, the associated boundary conditions are

$$
v_{0}=\theta_{x}=0 \quad \text { at } z=0
$$

and

$$
\left.\begin{array}{c}
a_{55}\left(v_{0}^{\prime}+\theta_{x}\right)+M \ddot{v}_{0}-M r_{M} \ddot{\theta}_{x}=0, \\
-- \\
a_{33} \theta_{x}^{\prime}+I_{M} \ddot{\theta}_{x}-M r_{M} \ddot{v}_{0}=\hat{M}_{x} . \\
--
\end{array}\right\} \quad \text { at } z=L .
$$

In these equations $v_{0}(z, t)$ and $\theta_{x}(z, t)$ denote the transverse deflection and rotation about the $x$ axis, respectively, $\delta(\cdot)$ denotes the Dirac's distribution, and $a_{33}$ and $a_{55}$ denote the bending and transverse shear stiffness, respectively. They are defined as

$$
\begin{aligned}
& a_{33}=\oint_{C}\left[\bar{K}_{11} y^{2}+\hat{K}_{11}\left(\frac{d x}{d s}\right)^{2}\right] d s, \\
& a_{55}=\oint_{C}\left[A_{66}\left(\frac{d y}{d s}\right)^{2}+A_{44}\left(\frac{d x}{d s}\right)^{2}\right] d s,
\end{aligned}
$$

where

$$
\begin{aligned}
& \bar{K}_{11}=A_{11}-\frac{A_{12}^{2}}{A_{11}}, \\
& \hat{K}_{11}=D_{11}
\end{aligned}
$$

while

$$
\left(A_{i j}, D_{i j}\right)=\sum_{k=1}^{N} \int_{n_{(k=1)}}^{n_{(k)}} C_{i j}^{(k)}\left(1, n^{2}\right) d n
$$

denote the stretching and bending stiffness quantities, respectively, while $N$ denotes the number of constituent layers.

In Eq. (6), $C_{i j}$ denotes the elastic coefficients, which, for the present type of anisotropy, are expressed in terms of the engineering constants as

$$
\begin{aligned}
& C_{11}=\left(E \nu^{\prime 2}-E^{\prime}\right) E / \Delta, \\
& C_{12}=-\left(E \nu^{\prime 2}+E^{\prime} \nu\right) E / \Delta, \\
& C_{13}=-\nu^{\prime}(1+\nu) E E^{\prime} / \Delta, \\
& C_{33}=-\left(1-\nu^{2}\right) E^{\prime 2} / \Delta, \\
& C_{44}=G^{\prime}, \\
& \frac{C_{11}-C_{12}}{2}=G\left(\equiv \frac{E}{2(1+\nu)}\right) .
\end{aligned}
$$

In these expressions $\Delta=(1+\nu)\left(2 E \nu^{\prime 2}+E^{\prime} \nu-E^{\prime}\right) ; E, \nu$, and $E^{\prime}, \nu^{\prime}$ denote Young's modulus and Poisson's ratio in the plane of isotropy and transverse to the plane of isotropy, respectively, while $G^{\prime}$ and $G$ denote the transverse shear and in-plane shear modulus, respectively.

In addition to these notations,

$$
\begin{aligned}
& \left(b_{1}, b_{4}\right)=\oint_{C} m_{0}\left(1, y^{2}\right) d s, \\
& b_{14}=\oint_{C} m_{2}\left[\frac{d x}{d s}\right]^{2} d s,
\end{aligned}
$$

stand for the mass terms, where

$$
\left(m_{0}, m_{2}\right)=\sum_{k=1}^{N} \int_{h_{(k-1)}}^{h_{(k)}} \rho_{(k)}\left(1, n^{2}\right) d n,
$$

$\rho$ denotes the mass density of the constituent materials, $p_{y}\left(\equiv p_{y}(z, t)\right)$ is the distributed transverse load (per unit span of the beam), while $r_{M}$ denotes the offset between the beam extremity and the centroid of the tip mass.

The primes denote derivatives with respect to the spanwise $z$ coordinate while the superposed dots denote derivatives with respect to time $t, \oint_{c}() d s$ denotes the integral around the circumference of the midline contour of the beam.

In the boundary condition [Eq. (3b)], $\hat{M}_{x}$ stands for the boundary moment control.

\section{SPECIAL CASE OF GOVERNING EQUATIONS}

The Bernoulli-Euler counterpart of the governing equations (1) and boundary conditions, Eqs. (2) and (3), is ob- 
tained through the elimination of $a_{55}\left(v_{0}^{\prime}+\theta_{x}^{\prime}\right)$ in Eqs. (1) and (3), operation followed by consideration of $\theta_{x}=-v_{0}^{\prime}$. In such a way, the classical equations governing the transverse bending of thin-walled beams carrying concentrated masses are

$$
\begin{gathered}
a_{33} v_{0}^{\prime \prime \prime \prime}-\left[\left(b_{4}+b_{14}\right) \ddot{v}_{0}^{\prime \prime}+\sum_{j=1}^{J} I_{j} \ddot{v}_{0}^{\prime \prime} \delta\left(z-z_{j}\right)\right] \\
+\left[b_{1} \ddot{v}_{0}+\sum_{j=1}^{J} m_{j} \ddot{v}_{0} \delta\left(z-z_{j}\right)\right]=p_{y},
\end{gathered}
$$

while the boundary conditions to be prescribed are

$$
v_{0}=v_{0}^{\prime}=0 \quad \text { at } z=0
$$

and

$$
\left.\begin{array}{c}
a_{33} v_{0}^{\prime \prime \prime}=M \ddot{v}_{0}+M r_{M} \ddot{v}_{0}^{\prime}, \\
-- \\
a_{33} v_{0}^{\prime \prime}-I_{M} \ddot{v}_{0}^{\prime}+M r_{M} \ddot{v}_{0}=\hat{M}_{x}
\end{array}\right\} \quad \text { at } z=L .
$$

It should be remarked that both the shearable and the classical nonshearable thin-walled beam models provide fourthorder governing equation systems (see Ref. 12).

In Eqs. (1) and (3) as well as in Eqs. (10) and (12), the terms identifying the rotatory inertia effect are underscored by a dotted line.

\section{THE CONTROL LAW}

The adaptive nature of the cantilevered beam is introduced by requiring that the applied electric field $\mathscr{E}_{3}$, or in other words the piezoelectrically induced bending moment at the beam tip, be related to one of the mechanical quantities characterizing its static or dynamic response. With this in mind, a number of feedback control laws featuring a static or a dynamic character have been implemented.

The static-type of control laws have been formulated by stating that the induced bending moment at the beam tip is proportional either with the mechanical bending moment at the beam root, or with the vertical deflection at the wing tip (see Refs. 9-14).

In contrast to the previously mentioned feedback control laws the one referred to as the velocity feedback control appears (see Refs. 7, 8, and 15) to be a good candidate for the problem at hand.

Within this control law, the moment $\hat{M}_{x}$ at the beam tip is proportional to the velocity $\dot{\theta}_{x}(L)$ at the same location. A more explicit expression of this feedback control law is derived from Eq. (3b) as

$$
\begin{aligned}
\theta_{x}^{\prime}(L) & +I_{M} /\left(a_{33}\right) \ddot{\theta}_{x}(L)+M r_{M} /\left(a_{33}\right) \ddot{v}_{0}(L) \\
= & \left(k_{v} / a_{33}\right) \dot{\theta}_{x}(L),
\end{aligned}
$$

where $k_{v}$ denotes the (dimensional) feedback gain.

In contrast to the previously mentioned control laws, the present one has a dynamic character and, as a result of it, damping is adaptively induced. Consequently, enhanced dynamic response performances are expected from its application.
Related with the bending moment at the beam tip, this can be generated via the use of the converse piezoelectric effect featured by these devices. In Refs. 7-13 it was shown that, in the case of piezoactuators spread over the entire beam span, polarized in the thickness direction, and featuring in-plane isotropy, under an applied electrical field $\mathscr{E}_{3}$, a bending moment at the beam tip is generated.

It should be mentioned that the piezoelectric induced moment $\hat{M}_{x}$ is different from zero only if external voltages, of opposite signs, are applied in the upper and bottom piezoactuator layers (out of phase activation).

For feedback control, the electric field $\mathscr{E}_{3}$ on which the induced bending moment depends should be related through a prescribed functional relationship with the mechanical quantities characterizing the wing's response. In the present case, the relationship is established with the rotational velocity at the beam tip.

\section{PROBLEMS STUDIED}

For the sake of numerical illustration, the case of a cylindrical thin-walled beam of a biconvex cross-section profile is adopted. The geometry of the cantilevered beam is shown in Fig. 1.

It is also considered, unless otherwise stated, that the beam carries a store of mass $m_{1}$ located along the beam span $\eta(\equiv z / L)$ and a tip mass $M$ as well. The beam mass is $m_{b}$ $\equiv b_{1} L$, where $b_{1}$ is the beam mass per unit length.

Within this paper two problems will be analyzed: the control of free vibration (problem I) and the dynamic response control to external excitation (problem II).

To analyze problem I, the external load term has to be discarded and all field variables, generically denoted as $F(z, t)$, have to be represented as

$$
F(z, t)=\bar{F}(z) \exp (\lambda t),
$$

where $\lambda$ is the closed-loop eigenvalue.

Since the eigenvalue is contained in both the governing equations and boundary conditions, it becomes apparent that the solution of the closed-loop eigenvalue and of that of the dynamic problems constitute a rather involved task.

The results obtained within the study of problem I are useful not only as a basic ingredient in the approach of problem II, but also in the study of the flutter instability of adaptive aircraft wings carrying external stores.

For problem II, one considers that the beam is excited by a concentrated harmonically time-dependent load located along the beam $z$ axis. Hence the load $p_{y}$ is represented as

$$
p_{y}(z, t)=F_{0} \delta\left(z-z_{0}\right) \exp (i \omega t)
$$

where $z_{0}, F_{0}$, and $\omega$ denote spanwise location of the load, and its amplitude and excitation frequency, respectively.

Both problems are solved via the extended Galerkin method (EGM) extensively used in Refs. 9-13.

As a matter of fact, the accuracy of this solution methodology was checked by comparing its predictions with the 


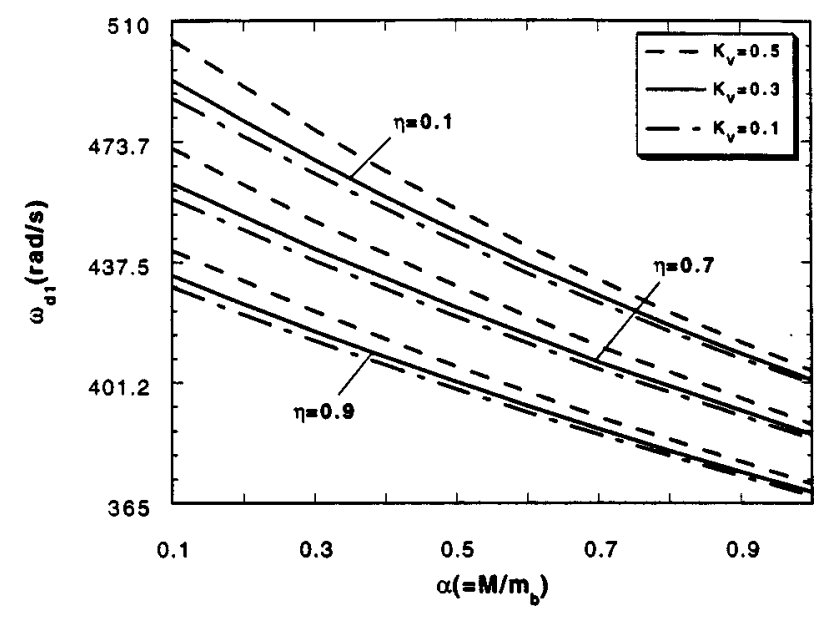

FIG. 2. The first closed-loop eigenfrequency versus the parameter $\alpha$, for three values of the feedback gain and of the position of the unistore along the beam span $\left(m_{1} / m_{b}=0.1\right)$.

ones based on an exact method, i.e., Laplace transform method (see Ref. 17) and the agreement was excellent. Once the frequency response functions corresponding to a given excitation frequency are determined [i.e., $v_{0}\left(z_{0}, \omega\right)$ and $\left.\theta_{x}\left(z_{0}, \omega\right)\right]$, their time-domain counterparts can be obtained as

$$
\begin{aligned}
\left\{\begin{array}{c}
v_{0}\left(z_{0}, t\right) \\
\theta_{x}\left(z_{0}, t\right)
\end{array}\right\} & =\mathscr{F}^{-1}\left\{\begin{array}{c}
v_{0}\left(z_{0}, \omega\right) \\
\theta_{x}\left(z_{0}, \omega\right)
\end{array}\right\} \\
& =\frac{1}{2 \pi} \int_{-\infty}^{+\infty}\left\{\begin{array}{c}
v_{0}\left(z_{0} \omega\right) \\
\theta_{x}\left(z_{0}, \omega\right)
\end{array}\right\} \exp (i \omega t) d \omega,
\end{aligned}
$$

where $\mathscr{F}^{-1}$ denotes the inverse Fourier transform.

The results of the study of problem II can constitute a good basis for a more reliable design of advanced structural systems as, e.g., aircraft wings or robot arm manipulators working in space which can carry stores and are exposed to external excitations.

\section{NUMERICAL APPLICATIONS AND DISCUSSION}

Within this section, numerical results pertaining to control of free vibration and dynamic response will be supplied and discussed.

\section{A. Free vibration control}

The closed-loop eigenfrequencies are obtained from Eqs. (1) and their associated boundary conditions Eqs. (2) and (3) considered in conjunction with the control law, Eq. (13). Within this problem the external load term is discarded and the field variables are represented in the form of Eq. (14). For the controlled structure, implying $k_{v} \neq 0$, the eigenvalues $\lambda$ are complex valued quantities expressible as

$$
\lambda=\sigma \pm i \omega_{d} .
$$

Based on their expressions one can determine the damped frequency $\omega_{d}$ and the damping ratio $\zeta$ defined as the imagi-

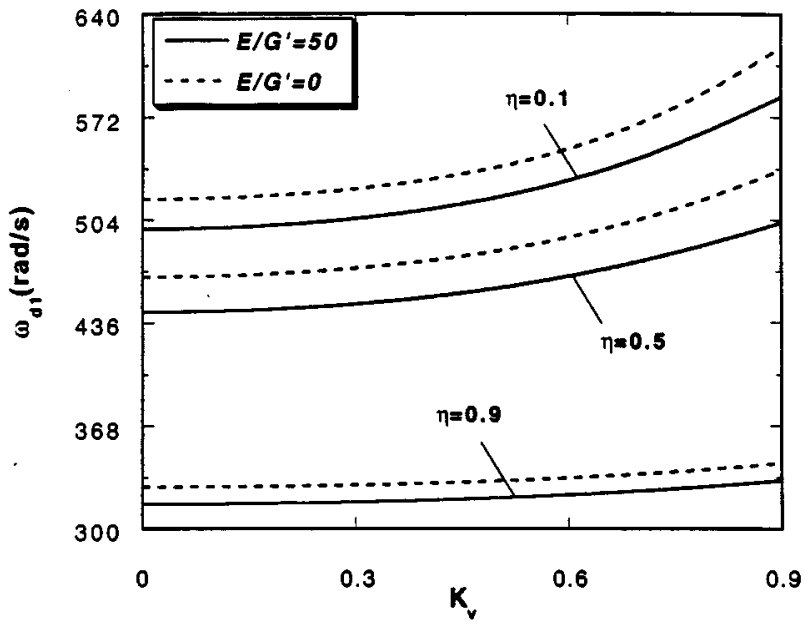

FIG. 3. The first closed-loop eigenfrequency versus the velocity feedback gain, for three positions of the unistore along the beam span and for the shearable and nonshearable beam. $(M=0, \mathrm{AR}=8)$.

nary and the negative of the normalized real part of Eq. (17), respectively, i.e.,

$$
\zeta=-\sigma /\left(\sigma^{2}+\omega_{d}^{2}\right)^{1 / 2} .
$$

Throughout the numerical illustrations, unless otherwise stated, it was considered that $E / G^{\prime}=50, M / m_{b}=0.1, r_{M}$ $=0.05 \mathrm{~L}, L=1 \mathrm{~m}, b=0.068 \mathrm{~m}$, and $c=0.25 \mathrm{~m}$. These dimensions correspond to a beam of aspect ratio, $\mathrm{AR}=8$ where $\mathrm{AR} \equiv 2 L / c$.

Figure 2 displays the influence of a tip mass characterized by the mass ratio $\alpha=M / m_{b}$ on the first closed-loop eigenfrequency of a shear deformable beam carrying an unistore at various locations $\eta(=z / L)$ along the beam span.

From this graph it becomes apparent that with the increase of the nondimensional feedback gain $K_{v}$ $\left(\equiv k_{v} L \bar{\omega} / a_{33}\right)$, the decay of the eigenfrequency resulting from the increase of $\alpha$ and location of the store towards the beam tip can be counteracted. At the same time, the results

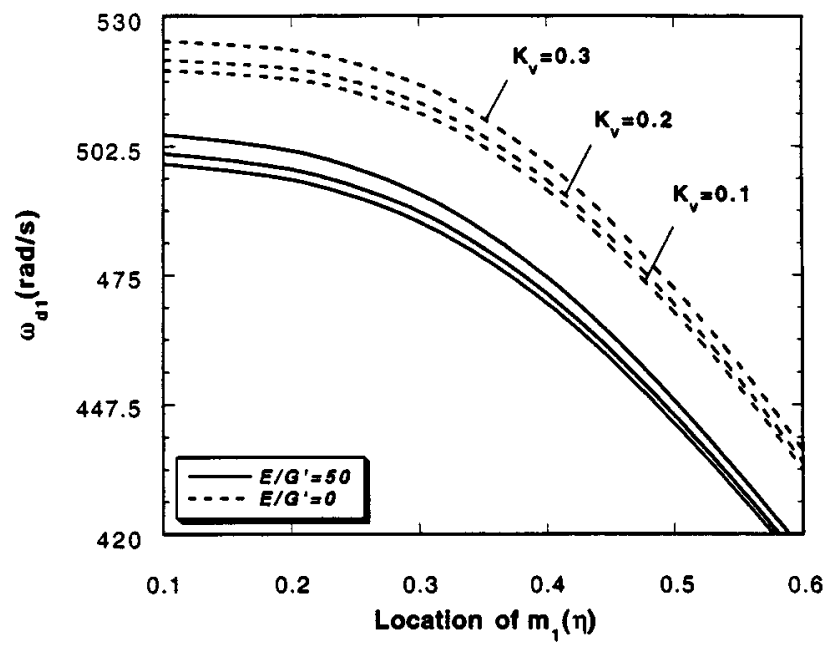

FIG. 4. The first closed-loop eigenfrequency versus the location $\eta$ of the unistore for the shearable and nonshearable and controlled and uncontrolled beam $\left(m_{1} / m_{b}=0.5, \mathrm{AR}=8, M=0\right)$. 


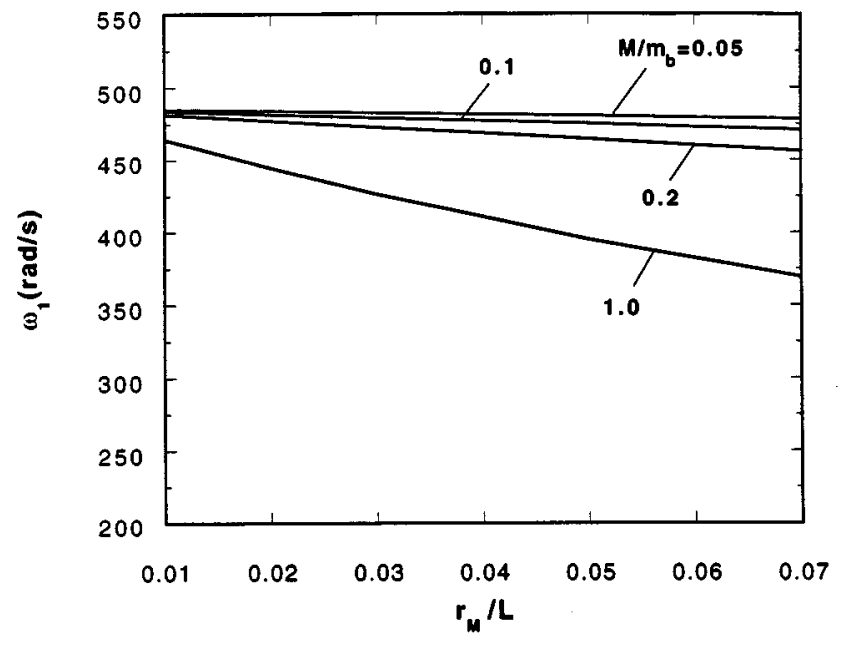

FIG. 5. The first open-loop eigenfrequency versus the dimensionless offset between the beam tip and the centroid of the tip-mass for four values of the ratio $M / m_{b}\left(E / G^{\prime}=50, m_{1} / m_{b}=0.1, \eta=0.5\right)$.

reveal that with the increase of $\alpha$, a decay in the efficiency of the control capability is experienced. Herein $\bar{\omega}(=128.16$ $\mathrm{rad} / \mathrm{s})$ is the fundamental eigenfrequency of the uncontrolled beam characterized by $E / G^{\prime}=50$ and $\mathrm{AR}=16$.

Figure 3 depicts the variation of the first damped eigenfrequency versus the feedback gain $K_{v}$, for three values of the location of the unistore and for the shearable and nonshearable beam models. The results reveal that: (a) as expected, the Bernoulli-Euler beam model (i.e., the model corresponding to $E / G^{\prime}=0$ ) overestimates the frequencies predicted by the actual shearable one and (b) the frequencies and control efficiency diminish with the increase of the distance of the store location, towards the beam tip.

The effect of location of the unistore along the beam span upon the closed-loop first eigenfrequency is highlighted in Fig. 4. Herein, the behavior resulting from the consideration of both shearable and nonshearable beam models are illustrated. The results presented in this figure enforce the previously obtained conclusions regarding the influence, on the closed-loop first eigenfrequencies, of transverse shear

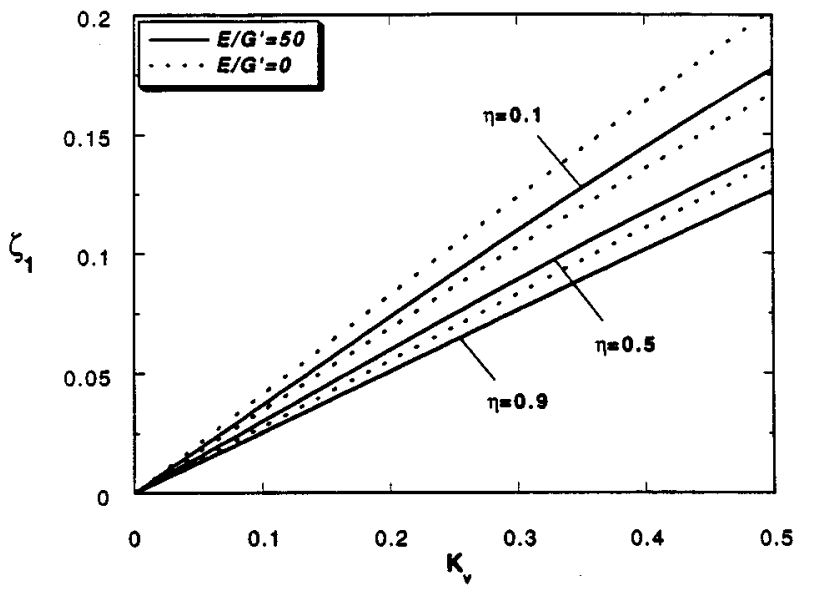

FIG. 6. Induced damping versus the feedback gain for three values of the unistore location and shearable and nonshearable beam $\left(m_{1} / b_{m}=0.5\right.$, AR $=8, M=0)$.

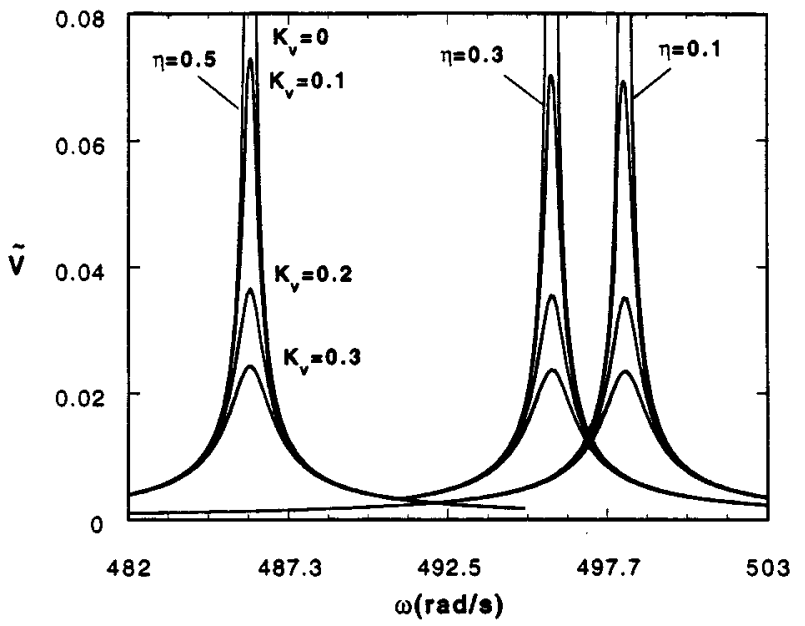

FIG. 7. Normalized steady-state deflection amplitude versus the excitation frequency for three locations of the unistore and for the controlled and uncontrolled beam $\left(m_{1} / m_{b}=0.1, M=0\right)$.

flexibility and position of the store along the beam span.

Figure 5 highlights the influence of the distance $r_{M}$ on the first eigenfrequency of the uncontrolled beam. The results obtained from this figure reveal that for smaller ratios $\mathrm{M} / \mathrm{m}_{b}$ the conclusions in Ref. 18 regarding the negligible influence of $r_{M}$ on the eigenfrequency are in perfect agreement with the present ones. However, with the increase of the ratio $M / m_{b}$, as is usual in space applications (see Ref. 19), this quantity can play a non-negligible influence on the decay of eigenfrequencies.

Figure 6 displays the variation of the first mode induced damping $\zeta_{1}$ as a function of the feedback gain in the case of a beam carrying a unistore located at different locations. The results reveal that: (a) the increase in the induced damping varies almost linearly with the increase of the feedback gain $K_{v}$, a trend which is consistent with that in Ref. 20; (b) as expected, the Bernoulli-Euler beam model overestimates the actual damping induced in the shear deformable beam coun-

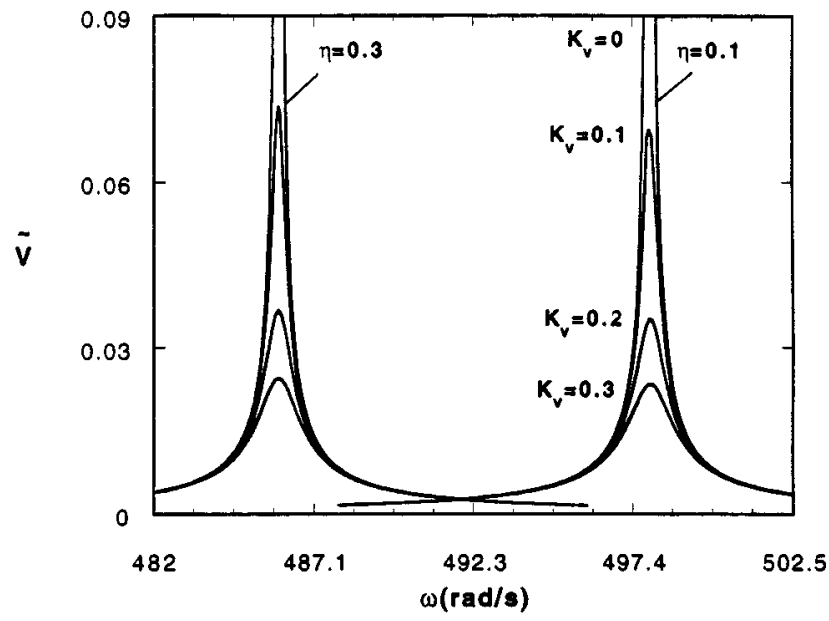

FIG. 8. Normalized steady-state deflection amplitude versus the excitation frequency for two locations of the unistore and for the controlled and uncontrolled beam $\left(M=0, m_{1} / m_{b}=0.5\right)$. 


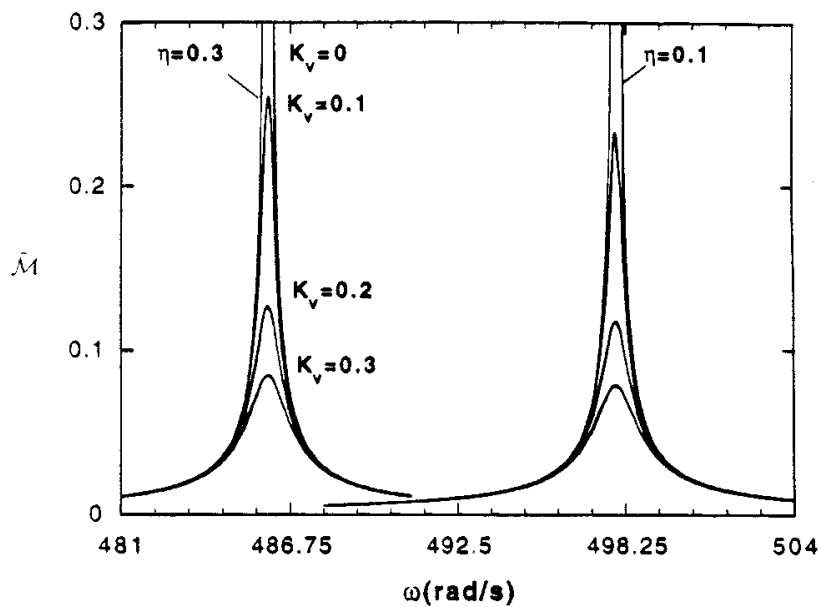

FIG. 9. Steady-state dimensionless root moment amplitude versus the excitation frequency for values of the location of the unistore $\left(m_{1} / m_{b}=0.5\right)$ and for the controlled and uncontrolled beam $(M=0)$.

terpart, overestimation which is exacerbated by the increase of $K_{v}$ and shift of the store toward the beam root.

\section{B. Dynamic response control}

Related with the dynamic response problem the variation of the dimensionless steady-state response amplitude $\widetilde{V}$ $\left(\equiv \bar{v}_{0} / L\right)$ of the beam tip, for the noncontrolled and controlled beam, as a function of the excitation frequency is depicted in Figs. 7 and 8, where the case of the unistore of $m_{1} / m_{b}=0.1$ and 0.5 , respectively, differently located along the beam span is considered.

In these graphs a shearable beam model is considered. The results reveal: (a) the capability of the control methodology to contain the level of vibration at the beam tip via the generation of damping, (b) the effect played by the location of the unistore along the beam span consisting of the shift of the resonance frequency towards larger frequencies when the unistore is located closer to the beam root, and, towards lower frequencies when the store is located closer to the beam tip. The results not displayed here reveal that the latter

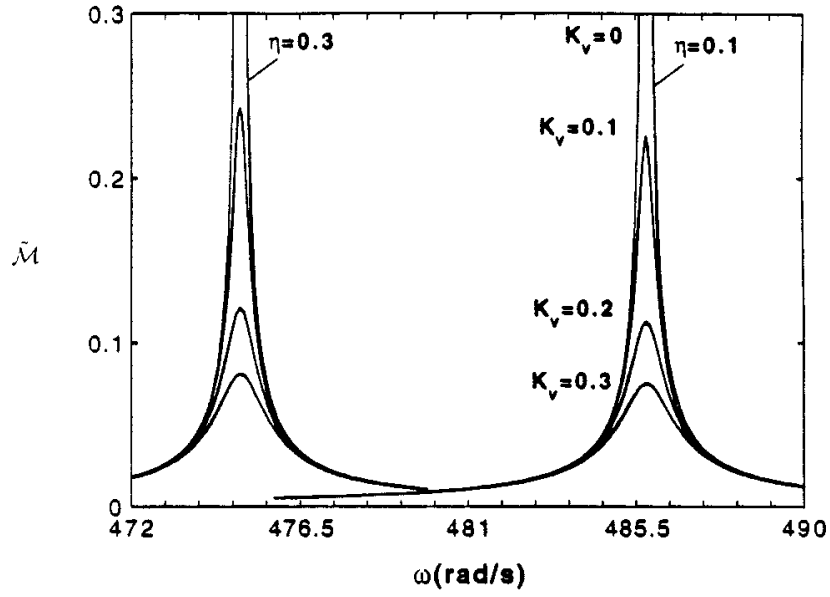

FIG. 10. The counterpart of Fig. 9 for the case when $M \neq 0$.

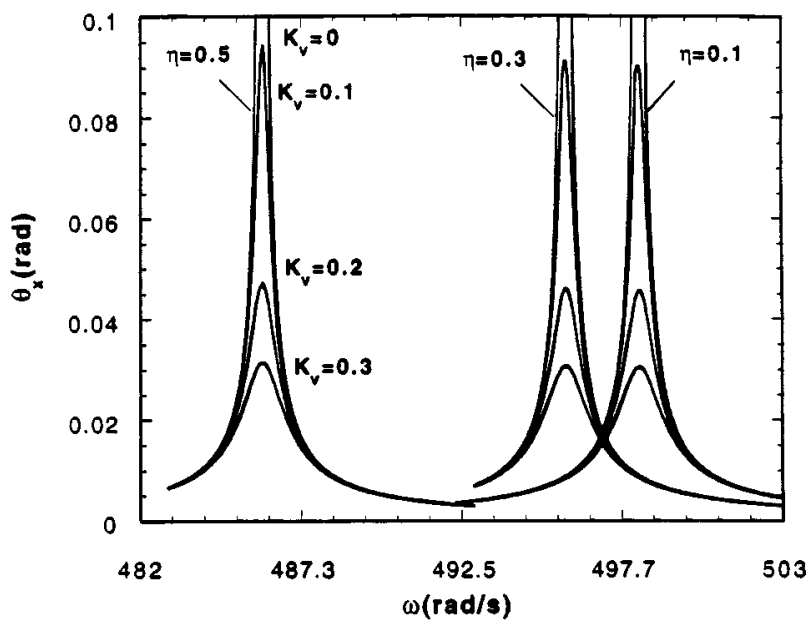

FIG. 11. Steady-state rotation amplitude versus the excitation frequency for three values of the location of the unistore $\left(m_{1} / m_{b}=0.1\right)$, and for controlled and uncontrolled beam $(M=0)$.

shift is further exacerbated when, in addition to the unistore, a tip mass is carried by the beam.

Figures 9 and 10 display the variation of steady-state dimensionless moment amplitude $\tilde{\mathscr{C}}\left(\equiv \theta_{x}^{\prime}(0)\right)$ at the beam root versus the excitation frequency. The results reveal that while the location along the beam span of the unistore has little effect upon the amplitude of the response, the shift towards lower or larger resonance frequencies (depending on the location of the store toward the beam tip or beam root, respectively) remains the most important effect in the context of the beam with attached stores or tip mass.

In this case, the shift of the resonance frequencies follows the same trend with respect to the ratio $m_{1} / m_{b}$ and the presence of the tip mass, as in the case of the dynamic response deflection amplitude (see Figs. 7 and 8). For the sake of completeness, Fig. 11 presents the variation of the steadystate rotation amplitude $\theta_{x}$ versus the excitation frequency. In this respect, similar conclusions to the ones highlighted by the behaviors of $\widetilde{V}$ and $\widetilde{\mathscr{C}}$ can be outlined in this case as well.

\section{CONCLUSIONS}

A control methodology carried out via the boundary moment and of a dynamic feedback control law aimed at enhancing the free vibration and dynamic response of cantilever beams carrying heavy concentrated masses was developed. The obtained results reveal that by using this control methodology, it is possible to modify in a beneficial and predictable way the vibration and dynamic response of these structures, as well to inhibit and even avoid, without weight penalties, the occurrence of the resonance phenomenon.

It should also be remarked that the control laws used in Refs. 9-13 and in the present paper are complementary to each other, in the sense that a combination of them can be used towards a more efficient static and dynamic control of such structures.

Finally, it should added that use of segmented piezoactuators, and specially of piezoelectric patches conveniently 
located, while resulting in a significant weight saving, would not yield a less control efficiency as compared to that provided by piezoactuators spread over the entire beam span. The preliminary results obtained so far, fully justify this statement.

${ }^{1}$ J. C. Bruch, Jr. and T. P. Mitchell, " Vibrations of a mass-loaded clampedfree Timoshenko beam," J. Sound Vib. 114, 341-345 (1987).

${ }^{2} \mathrm{H}$. Abramovich and O. Hamburger, "A vibration of a cantilever Timoshenko beam with a tip mass," J. Sound Vib. 148, 162-170 (1991).

${ }^{3}$ W. E. Triplett, H. P. F. Kappus, and R. J. Landy, "Active flutter control-An adaptable application to wing/store flutter," J. Aircr. 10(11), 669-678 (1973).

${ }^{4} \mathrm{H}$. Zimmerman and S. Vogel, "Influence of main design parameters on flutter behavior of large aircraft configurations with heavy concentrated masses," Report No. AGARD-CP-354, pp. 6-1 to 6-11 (1983).

${ }^{5}$ J. E. Lagnese and J. L. Lions, "Boundary stabilization of thin plates," in Collection RMA (Masson, Paris, 1988).

${ }^{6}$ J. E. Lagnese, "Boundary stabilization of thin plates," SIAM Studies in Applied Mechanics (SIAM, Philadelphia, 1989).

${ }^{7}$ T. Bailey and J. E. Hubbard, Jr., "Distributed piezoelectric-polymer active vibration control of a cantilever beam" J. Guid. Control. Dyn. 8, 605-611 (1985).

${ }^{8}$ H. S. Tzou, Piezoelectric Shells, Distributed Sensing and Control of Continua (Kluwer Academic, Dordrecht, 1993).

${ }^{9}$ O. Song, L. Librescu, and C. A. Rogers, "Application of adaptive technology to static aeroelastic control of wing structures," AIAA J. 30, 2882-2889 (1992).

${ }^{10}$ L. Librescu, O. Song, and C. A. Rogers, "Adaptive vibrational behavior of cantilevered structures modeled as composite thin-walled beams," Int. J. Eng. Sci. 31, 775-792 (1993).

${ }^{11}$ O. Song, L. Librescu, and C. A. Rogers, "Adaptive response control of cantilevered thin-walled beams carrying heavy concentrated masses," J. Intell. Mater. Syst. Struct. 5, 42-48 (1994).

${ }^{12} \mathrm{O}$. Song and L. Librescu, "Bending vibrations of adaptive cantilevers with external stores," Int. J. Mech. Sci. 38, 483-498 (1996).

${ }^{13}$ L. Librescu, L. Meirovitch, and O. Song, "Integrated structural tailoring and adaptive materials control for advanced aircraft wings," J. Aircr. 33, 203-213 (1996).

${ }^{14}$ H. S. Tzou and J. R. Zhong, "Adaptive piezoelectric structures: Theory and experiment," Active Materials and Adaptive Structures, Proceedings of the ADPA/AIAA/ASME/APIE Conference on Active Materials and Adaptive Structures, Alexandria, VA, edited by G. J. Knowles, pp. 719724.

${ }^{15} \mathrm{~A}$. Baz, "Boundary control of beams using active constrained layer damping," J. Vib. Acoust., Trans. ASME 9, 166-172 (1997).

${ }^{16}$ A. Gjelsvik, The Theory of Thin Walled Bars (Wiley, New York, 1981).

${ }^{17} \mathrm{O}$. Song and L. Librescu, "Bending vibration of cantilevered thin-walled beams subjected to time-dependent external excitations," J. Acoust. Soc. Am. 97, 313-319 (1995).

${ }^{18}$ M. W. D. White and G. R. Heppler, "Dynamics of Timoshenko beams with attached masses," Dynamics and Control of Structures in Space II, edited by C. L. Kirk and R. C. Hughes (Computational Mechanics, Southampton, Boston, 1993), pp. 209-224.

${ }^{19}$ R. Kumar, "Vibrations of space booms under centrifugal force field," Can. Aeronaut. Space Inst. (CASI) Trans. 7, 1-6 (1974).

${ }^{20}$ H. S. Tzou, J. P. Zhong, and J. J. Hollkamp, "Spatially distributed orthogonal piezo-electric shells actuators: Theory and applications" J. Sound Vib. 177, 363-378 (1994) 